\title{
Role of DOR in neuronal plasticity changes promoted by food-seeking behaviour.
}

S. Mancino ${ }^{1}$, S. Mendonça Netto ${ }^{1}$, E. Martín-García ${ }^{1}$, R. Maldonado ${ }^{1, *}$

${ }^{1}$ Departament de Ciencies Experimentals i de la Salut, Universitat Pompeu Fabra, Barcelona, Spain.

* Correspondence should be addressed to Rafael Maldonado:

Dr. Aiguader, 8808003 Barcelona

rafael.maldonado@upf.edu

Phone: +34-93-3160824

Fax: +34-93-3160901

Category of the article: Regular research article

Number of references: 66

Words in Abstract: 241

Words in Article (excluding abstract, references and figure legends): 5344

Number of figures: 4

Number of tables: 2

Short title: The involvement of DOR in food-seeking behaviour 


\section{Abstract}

Several lines of evidence support that food overconsumption may be related to the role of the endogenous opioid system in the control of food palatability. The opioid system, and particularly the delta opioid receptor (DOR), plays a crucial role in the regulation of food rewarding properties. In our study, we used operant conditioning maintained by chocolate-flavoured pellets to investigate the role of DOR in the motivation for palatable food and the structural plasticity changes promoted by this behaviour. For this purpose, we evaluated the specific role of this receptor in the behavioural and neuroplastic changes induced by palatable food in the prefrontal cortex (PFC), hippocampus (HCP) and nucleus accumbens (NAc) in constitutive knockout (KO) mice deficient in DOR. Mutant mice and their wild-type littermates were trained to obtain chocolate-flavoured pellets on fixed ratio 1 (FR1), FR5 and progressive ratio (PR) schedule of reinforcement. No significant differences between genotypes were revealed on operant behaviour acquisition in FR1. DOR knockout mice displayed lower number of active lever-presses than wild-type mice on FR5, and a similar decrease was revealed in DOR KO mice in the breaking point during the PR. This operant training to obtain palatable food increased dendritic spine density in the PFC, HCP and NAc shell of wildtype, but these plasticity changes were abolished in DOR KO mice. Our results support the hypothesis that DOR regulates the reinforcing effects and motivation for palatable food through neuroplastic changes in specific brain reward areas.

Key Words: compulsive eating, motivation, operant behaviour, neuroplasticity. 


\section{Introduction}

Obesity and overeating are among the most important health issues affecting the developed countries (Hill, 2006). Genetic predisposition and environmental influences are two prominent risk factors related to these disorders. Obesogenic environment and the high availability of palatable foods contribute to the present high rate of obesity. It is estimated that about $13 \%$ of the world's adult population $(11 \%$ of men and $15 \%$ of women) was obese in 2014 and this percentage will tend to increase in the next years (World Health Organization WHO, 2015).

Palatability is an important determinant of the reward value of food that can promote the overconsumption leading to overweight and obesity. The pleasant experience obtained from palatable food contributes to repeat the experience and can result in the development of loss of control over intake (Kenny, 2011). Food, like drugs, activates the mesocorticolimbic system involved in the hedonic effect promoted by different rewards and may produce neuroadaptive responses in these circuitries by mechanisms that resemble drugs of abuse (Volkow et al., 2013).

The involvement of the opioid system in food hedonic effects has been known for several decades (Yeomans and Gray, 2002). The opioid system is integrated by three different opioid receptors, mu receptor (MOR), delta receptor (DOR) and kappa receptor (KOR), widely distributed in the central nervous system and located in brain areas mediating food intake and reward (Mansour et al., 1995). Rewarding effects are mainly mediated by MOR and DOR, which activation increases dopamine (DA) release in the nucleus accumbens (NAc) shell (Hirose et al., 2005; Okutsu et al., 2006) by decreasing GABA-inhibition in the ventral tegmental area (VTA) (Bonci and Williams, 1997). MOR and DOR are also involved in the inhibitory control process in cortical 
areas, whose dysregulation is associated with addiction and eating disorders (Mitchell et al., 2007; Selleck et al., 2015). Although the involvement of MOR in eating disorders (Ziauddeen et al., 2013) and obesity (Tabarin et al., 2005) is largely demonstrated, little is known about the potential implication of DOR in these diseases.

Behavioural alterations induced by rewarding stimuli might be triggered by plasticity adaptations in neuronal circuitries that seem to be mediated by the opioid system (Mazei-Robison and Nestler, 2012). Indeed, this system is implicated in cellular and synaptic plasticity through the regulation of glutamatergic and GABAergic transmission (Luscher and Malenka, 2011), and structural plasticity through long-lasting changes of neuronal morphology (Russo et al., 2010). Specifically, DOR seems directly involved in long term depression (LTD) in hippocampus (HCP) (Piskorowski and Chevaleyre, 2013) and it is also required to induce long term potentiation (LTP) in the lateral perforant path (Bramham and Sarvey, 1996). However, the specific implication of DOR in structural plasticity changes has not been clarified. Neuronal and dendritic spine morphogenesis underlie experience-dependent brain development and information storage (Holtmaat and Svoboda, 2009). Dendritic spines are highly dynamic structures, and their stabilization and morphology are influenced by synaptic activity (Lai and Ip, 2013). An increase in synaptic strength by induction of LTP is generally associated with the enlargement of pre-existing spines, the stabilization of newly-formed spines, and the formation of new spines increasing the connectivity of neurons (De Roo et al., 2008). In contrast, a reduction of synaptic strength during the LTD is correlated with spine shrinkage and retraction (Lai and Ip, 2013). A high correlation exists between the physiology of synaptic transmission and the shape of the dendritic spine and both phenomena could play complementary functions in neuronal plasticity (Bosch and Hayashi, 2012). 
In this study, we examine the role of DOR in the motivational properties and morphological plasticity changes induced by palatable food in prefrontal cortex (PFC), HCP and NAc core and shell. These regions are crucial for regulating food hedonic aspects and dysfunctions of these areas have been related to eating disorders (Kelley and Berridge, 2002; Volkow et al., 2008). The NAc shell participates in the hedonic impact for rewards (Bodnar et al., 2005), the NAc core in response-reinforcement learning (Di Ciano and Everitt, 2001), the PFC in decision-making and inhibitory control processes (Volkow et al., 2008) and the HCP in memory processes that regulate motivational salient events (Kahn and Shohamy, 2013). 


\section{Materials and methods}

\section{Animals}

Homozygous knockout (KO) mice deficient in DOR on a C57BL/6J background and their respective wild-type littermates were used (Filliol et al., 2000). A different group of DOR KO mice and wild-type naïve mice of the same age and under the same housing conditions not trained with chocolate pellets was added as a control for the synaptic plasticity experiment. Mice weighted $31 \pm 1.5 \mathrm{~g}$ at the beginning of the experiment. Mice were housed individually in controlled laboratory conditions with the temperature maintained at $21 \pm 1^{\circ} \mathrm{C}$ and humidity at $55 \pm 10 \%$, and were tested during the first hours of the dark phase of a reversed light/dark cycle (lights off at 8.00 a.m. and on at 8:00 p.m.). Food and water were available ad libitum to avoid confounding factors linked to deprivation experience (Piazza and Le Moal, 1998). Animal procedures were conducted in strict accordance with the guidelines of the European Communities Directive 86/609/EEC regulating animal research and were approved by the local ethical committee (CEEA-PRBB).

\section{Operant behaviour apparatus}

Operant responding maintained by food was performed in mouse operant chambers (Model ENV-307A-CT, Med Associates, Georgia, VT, USA) equipped with two retractable levers, one randomly selected as the active lever and the other as the inactive, as previously described (Martín-García et al., 2011). Pressing on the active lever resulted in a pellet delivery together with a stimulus-light located above the active lever. Pressing on the inactive lever had no consequences. The chambers were housed in sound and light-attenuated boxes equipped with fans to provide ventilation and white 
noise. A food dispenser equidistant between the two levers permitted the delivery of food pellets when required.

\section{Food pellets}

During the operant experimental sessions, animals received after each active responding a $20 \mathrm{mg}$ highly palatable isocaloric pellet (20.5\% proteins, $12.7 \%$ fats and $66.8 \%$ carbohydrates, with a caloric value of $3.48 \mathrm{kcal} / \mathrm{g}$; TestDiet, Richmond, IN, USA) with similar caloric value than the maintenance diet provided for mice in their home cage (24.1\% proteins, $10.4 \%$ fats and $65.5 \%$ carbohydrates, with a caloric value of 3.30 kcal/g; Diets Services, Witham, Essex UK) with some slight modifications in its composition: addition of chocolate flavour ( $2 \%$ pure unsweetened cocoa) and modification in the sucrose content. Indeed, although the carbohydrate content was similar in maintenance diet (65.5\%) and highly palatable isocaloric pellets (66.8\%), the sucrose content in standard chow was $3.1 \%$ of the total carbohydrates and $50.1 \%$ in highly palatable isocaloric pellets. These pellets were presented only during the $1 \mathrm{~h}$ daily operant session and animals were maintained on standard chow for their daily food intake.

\section{Experimental design}

DOR KO mice and their wild-type littermates were trained in operant boxes during $1 \mathrm{~h}$ daily sessions to acquire operant responding maintained by chocolate-flavoured pellets under FR1 during 16 consecutive days and FR5 during 95 consecutive days. A stimulus light, located above the active lever, was paired contingently with the delivery of the reward during the active periods. The beginning of each operant responding session was signalled by turning on a house light placed on the ceiling of the box only during the 
first $3 \mathrm{sec}$ of the session. A time-out period of $10 \mathrm{sec}$ was established after each pellet delivery. During this period, the cue light was off and no reinforcer was provided after responding on the active lever. Responses on the active lever and all the responses performed during the time-out period were recorded. The criteria for acquisition of operant responding were achieved when mice maintained a stable responding with less than $20 \%$ deviation from the mean of the total number of food pellets earned in three consecutive sessions, with at least $75 \%$ responding on the reinforced lever, and a minimum of 10 reinforcers per session (Martín-García et al., 2011). Motivation for food was evaluated in each mouse at day 70 of the operant training using the progressive ratio (PR) schedule where the response required to earn the pellet escalated according to the following series: $1,5,12,21,33,51,75,90,120,155,180,225,260,300,350,410$, 465, 540, 630, 730, 850, 1000, 1200, 1500, 1800, 2100, 2400, 2700, 3000, 3400, 3800, $4200,4600,5000,5500$. The maximal number of responses that the animal performs to obtain one pellet is the last ratio completed, referred to the breaking point. The maximum duration of the PR session was 5 h or until mice did not respond on any lever within $1 \mathrm{~h}$.

\section{Sample preparation}

Immediately after the last training session, mice were deeply anesthetized by intraperitoneal injection $(0.2 \mathrm{ml} / 10 \mathrm{~g}$ body weight) of a mixture of ketamine (100 $\mathrm{mg} / \mathrm{kg}$ ) and xylazine (20 mg/kg) prior to rapid intracardiac perfusion, delivered with a peristaltic pump with $10 \mathrm{ml}$ of Na2HPO4/NaH2PO4/NaCl buffer (PBS) 0.1M, pH 7.5, and followed by perfusion with $40 \mathrm{ml}$ of $4 \%$ paraformaldehyde (PFA) in PBS 0.1M, pH 7.5. Brains were quickly removed from the skull and post fixed in 4\% PFA for $10 \mathrm{~min}$. Brain coronal sections $(100 \mu \mathrm{m})$ containing the NAc (from bregma, AP: from 1.54 to 
$0.98 \mathrm{~mm}$; ML: $\pm 0.5 \mathrm{~mm}$; DV: - $4 \mathrm{~mm}$ ), medial PFC (mPFC) (from bregma, AP: from 2.10 to $1.70 \mathrm{~mm}$; ML: $\pm 0 \mathrm{~mm}$; DV: $-3.5 \mathrm{~mm}$ ) and HCP (from bregma, AP: from 1.58 to $2.06 \mathrm{~mm}$; ML: $\pm 0 \mathrm{~mm}$; DV: $-2.8 \mathrm{~mm}$ ) (Paxinos and Franklin, 1997) were obtained by using a vibratome (Leica VT 1000 S, Nussloch, Germany) and kept in PBS 0.1 M until they were processed for fluorescent labelling.

\section{Dendritic spine analysis}

Brain slices were labelled by ballistic delivery of fluorescent dye DiI (Molecular Probes, Eugene, OR, USA) using a gene gun apparatus (Helios Gene Gun System, BioRad, Deutschland), as already reported (Grutzendler et al., 2003), and post fixed with PFA overnight at room temperature to further preserve structures and to allow the diffusion of the dye DiI. Brain sections were placed on microscope gelatine coated slides and cover slipped with mounting medium (Mowiol). Then, images were acquired with confocal microscope (Zeiss LSM 510, Germany) with an oil immersion lens (63x) to analyze dendritic spine density and structure.

Individual pyramidal neurons from the mPFC and HCP, and medium spiny neurons in the NAc were chosen for spine analysis based on several criteria, as described previously (Lee et al., 2006): (i) there was minimal or no overlap with other labelled cells, (ii) at least three primary dendrites needed to be visible for cells to be used for analysis, and (iii) distal dendrites (from secondary dendrites to terminal dendrites) were examined. Dendrites from medium spiny neurons in the core and shell of the NAc, basilar dendrites of pyramidal neurons taken predominantly from the prelimbic and infralimbic areas of the mPFC, usually CA1 and CA3 dendrites of pyramidal neurons of HCP were analyzed. A total of 3 to 8 mice per experimental group were used and 4 to 11 dendrites per brain area and mouse were analyzed (max two dendrites/neuron). 
To calculate spine density, a length of dendrite (at least $21 \mu \mathrm{m}$ long) was traced. All images of dendrites were taken at different z levels (0.3 $\mu \mathrm{m}$ depth intervals) to examine the morphology of dendritic spines. Before the analysis, all images were processed with the deconvolution software (Huygens Essential, The Netherlands) that reduces the optical noise generated by the contribution of out-of-focus fluorescent points and by the distortion due to the optical instrument. All measurements were made using IMARIS 7 analysis software (Bitplane scientific software, Swiss) with the aim of automating dendritic spine analysis. Protrusions from dendrites were classified into four types based on their morphology pursuant to a logarithm program that sorts each spine according to parameters as the length spine, the width neck and the width head. After visual identification of stubby and headed spines, parameters defined by the program identify and distinguish the spine length against spine head size, and spine minimum diameter against spine head size. Stubby spines stand out from headed spines by the ratio spine minimum diameter/spine head diameter, which was equal to $1 \mu \mathrm{m}$ for a majority of stubby spines (94\%), and less than $1 \mu \mathrm{m}$ for spines with an identifiable neck. After finding an efficient way of sorting stubby spines, the program defines parameters to sort out large spines. Head size was the first obvious parameter. Accordingly, mushroom spines were classified as having width head greater than width neck multiplied by 2 . The remaining spines could be separated on their neck length to head size ratio as thin, if the length spine was $>3 \mu \mathrm{m}$, and the width head was greater than the width neck, or filopodia spines, if the length was $>3 \mu \mathrm{m}$ and the width head was equal to the width neck. 


\section{Statistical analysis}

Data obtained during the operant acquisition phase were analysed using three-way repeated measures analysis of variance (ANOVA) with manipulandum (active/inactive) and day as within-subjects factors and genotype as between-subjects factor. PR and body weight data were analysed using one-way ANOVA between subjects (genotype). Time-out period was analysed using Mann-Whitney $U$ due to the non-normally distributed data according to Kolmogorov test (Supporting Information Table S1A). Structural plasticity data were analysed using one-way ANOVA with group as betweensubjects factor (naïve wild-type, naïve DOR knockout mice (DOR KO), trained DOR KO and trained wild-type littermate mice). Post-hoc analysis (Newman-Keuls or Dunnet's) was performed when required. All the results are expressed as mean \pm S.E.M. Differences were considered significant at $\mathrm{p}<0.05$. The statistical analysis was performed using the Statistical Package for Social Science program SPSS ${ }^{\circledR} 19.0$ (SPSS Inc, Chicago, USA). 


\section{Results}

\section{Acquisition of operant behaviour to obtain chocolate flavoured-pellets in DOR and}

\section{wild-type littermate mice.}

DOR KO and wild-type littermate mice were trained to acquire an operant responding maintained by chocolate flavoured-pellets under FR1 and FR5 schedule. The acquisition criteria in FR1 were achieved after an average of $11.3 \pm 1.03$ sessions by $28.6 \%$ of the entire DOR KO group and of $12.6 \pm 1.03$ sessions by $66.7 \%$ of wild-type littermates. Chi square test revealed significant differences in the percentage of acquisition criteria $(\chi 2=9.14, \mathrm{p}<0.05)$. Three-way ANOVA revealed a significant main effects of genotype, lever and day indicating a continuous operant responding for chocolate pellets during the whole training period and discrimination between levers $[F(1,24)=44.52$, $p$ $<0.001]$ (Figure 1). No significant genotype differences were revealed (Table 1).

The acquisition criteria in FR5 were achieved after an average of $8.55 \pm 5.06$ sessions by $78.6 \%$ of the entire DOR KO group and of $6.08 \pm 0.71$ sessions by $100 \%$ of wildtype littermates. Three-way ANOVA revealed significant main effects of lever $[F(1,24)$ $=125.85, \mathrm{p}<0.001]$ indicating discrimination in the two groups, and an interaction between genotype and lever $[\mathrm{F}(1,24)=16.95, \mathrm{p}<0.001]$, indicating higher number of active lever-presses for wild-type littermates (Figure 1) (Table 1). Motivation for food measured at day 70 on the PR schedule showed a significant decrease in DOR KO mice when compared with wild-type littermates [one-way ANOVA, $F(1,24)=1.03$, $p<0.05$ ] (Figure 2A). The number of lever-presses during the time-out period was also evaluated as a measurement of impulsivity like-behaviour and decreased active lever-presses during FR1 and FR5 training sessions were observed in DOR KO when compared with wild-type mice [Mann-Whitney test, $\mathrm{p}<0.05$ and $\mathrm{p}<0.01$, respectively] (Figure 2B). 


\section{Body weight}

No differences in body weight were found between genotypes during the FR1 training. As expected, a significant increase of body weight was reported during the FR5 training period when compared to the previous FR1 period in DOR KO mice and their wild-type littermates [one-way ANOVA, $\mathrm{F}(1,26)=25.87, \mathrm{p}<0.001 ; \mathrm{F}(1,22)=18.32, \mathrm{p}<0.001$ ]. In contrast to the FR1 period, significant differences were revealed between DOR KO mice and their wild-type littermates during FR5 training [one-way ANOVA, F(1,24) = 4.30, $\mathrm{p}<0.05$ ] (Figure 2C).

\section{Operant behaviour to obtain chocolate flavoured-pellets modifies structural plasticity in DOR KO and wild-type littermates.}

Changes in dendritic spine density were analysed with ballistic labelling with the fluorescent dye Dil in the PFC, HCP, NAc shell and NAc core. Significant differences in total dendritic spine density in neurons from the PFC [one-way ANOVA, F(3,19) = 13.89, $\mathrm{p}<0.001$ ], HCP [one-way ANOVA, $\mathrm{F}(3,13)=5.70, \mathrm{p}<0.05$ ] and NAc shell [one-way ANOVA, F(3,17) = 23.51, $\mathrm{p}<0.001$ ] were reported. Newman-Keuls post-hoc test revealed significant differences between wild-type mice trained with chocolate flavoured pellets and naïve wild-type mice $(\mathrm{p}<0.05)$ and between trained wild-type mice and DOR KO mice also trained with these pellets ( $\mathrm{p}<0.001)$. In addition, significant differences in total dendritic spine density were reported between wild-type mice trained with chocolate flavoured pellets and naïve DOR KO mice in neurons from the PFC ( $p<0.01)$, HPC $(p<0.05)$ and NAc Shell $(\mathrm{p}<0.001)$. Significant differences in NAc Shell were also found in naïve DOR KO mice when compared to naïve wildtype mice and DOR KO trained mice $(\mathrm{p}<0.001)$. However, no differences were found 
between groups in the NAc core [one-way ANOVA, F(3,14) $=1.67$, n.s.] (Figure 3A,B).

Changes in specific types of dendritic spines were also analysed in each brain area (see table 2 for one-way ANOVA). Newman-Keuls post-hoc test revealed significant differences between groups. In the PFC, significant differences in wild-type mice trained with chocolate pellets when compared to wild-type naïve mice were reported in thin $(\mathrm{p}<0.01)$, mushroom $(\mathrm{p}<0.05)$ and filopodia $(\mathrm{p}<0.01)$ density. In addition, significant differences were also reported between DOR KO mice non-trained and wildtype mice trained with chocolate pellets in thin $(\mathrm{p}<0.001)$, mushroom $(\mathrm{p}<0.05)$ and filopodia $(\mathrm{p}<0.01)$ density. Significant differences were also reported in stubby $(\mathrm{p}<$ 0.05), thin $(\mathrm{p}<0.01)$, mushroom $(\mathrm{p}<0.05)$ and filopodia $(\mathrm{p}<0.01)$ density between wild-type and DOR KO mice trained with these pellets. In addition, decreased stubby and filopodia spine density was reported in DOR KO when compared with naïve wildtype mice $(\mathrm{p}<0.05)$. No major changes were found in stubby, mushroom and filopodia spine density in DOR KO trained mice when compared to DOR KO non-trained group, although an increased density of thin spines were revealed in DOR KO trained mice with respect to DOR KO non-trained mice $(\mathrm{p}<0.05)$ (Figure 4A).

In the HCP, an increase in the density of stubby spines was shown in wild-type mice trained with chocolate pellets when compared with naïve wild-type mice ( $\mathrm{p}<0.01)$, DOR KO mice trained $(\mathrm{p}<0.01)$ and non-trained with these pellets $(\mathrm{p}<0.01)$. No significant changes in the different types of spine density were observed between naïve DOR KO mice and DOR KO mice trained with chocolate pellets although a decrease in filopodia spine density was observed in DOR KO trained mice compared to wild-type naïve (p < 0.05) (Figure 4B). 
In the NAc shell, an increase of filopodia density was observed in wild-type mice trained with chocolate pellets with respect to the naïve wild-type group ( $\mathrm{p}<0.001$ ) and DOR KO mice trained $(\mathrm{p}<0.01)$ and non-trained with these pellets $(\mathrm{p}<0.001)$. No significant modifications in the different types of spine density were observed between naïve wild-type mice and DOR KO mice trained with chocolate pellets, as well as between DOR KO trained mice and DOR KO non-trained group. Decreased stubby spine density was found in DOR KO non-trained mice when compared to the wild-type trained $(\mathrm{p}<0.01)$ and non-trained groups $(\mathrm{p}<0.01)$ (Figure 4C).

No major modifications were reported among groups in the NAc core, although increased mushroom spine density was revealed in DOR KO mice trained with chocolate pellets when compared with naïve DOR KO mice non-trained group ( $\mathrm{p}<$ 0.05) (Figure 4D). 


\section{Discussion}

The present study reveals a novel role of DOR in mediating the reinforcing effects, motivation and impulsive-like behaviour induced by operant training maintained with chocolate flavoured-pellets. In addition, prolonged operant training to obtain palatable food modifies structural plasticity in the PFC, HCP and NAc shell of DOR KO mice and their wild-type littermates, which suggests a specific involvement of DOR in the plasticity changes promoted by this repeated palatable food operant training.

Our results reveal a critical role of DOR in mediating the reinforcing aspects of foodseeking behaviour. During the FR1 schedule of reinforcement, only $28 \%$ of DOR KO mice achieved the acquisition criteria in contrast to $66 \%$ of wild-type mice. These results provide an initial evidence of the role of DOR in the acquisition of this operant behaviour. The two genotypes showed similar increases in food operant responding across the FR1 period suggesting preserved cognitive/learning abilities to acquire the food-maintained operant task in DOR-deficient mice. A similar performance in operantresponding maintained by high-fat food was already previously reported in fooddeprived mice, which facilitates operant responding (Gutierrez-Cuesta et al., 2014). These findings ruled out a potential learning impairment for operant training in these knockout mice. To avoid possible confounding factors, the role of DOR in the reinforcing and motivational properties of food consumption was examined in the present experimental conditions in mice trained with chocolate pellets and fed ad libitum in their home cage, since restrict access to food induces major changes in the brain opioid system (Wolinsky et al., 1994). During the FR5 schedule, DOR KO mice showed less operant responding than wild-type mice as revealed by the decreased number of active lever-presses during the whole training period. Similar reduction of 
the operant responding was observed for DOR KO mice during the PR schedule. The breaking point is a sensible and validated measure of the motivational state of the animal (Arnold and Roberts, 1997). Therefore, the present result suggests that disruption of DOR reduced the motivation for chocolate-flavoured pellets consumption. Previous studies have also suggested that DOR participates in the rewarding aspects associated with preferred foods (Gosnell and Majchrzak, 1989), according with the decreased motivation toward chocolate pellets of DOR KO animals in our experimental conditions. In agreement, central administration of DOR agonists increased saccharin solution intake in rats and this increase was selective for saccharin, since water intake was minimal (Gosnell and Majchrzak, 1989). Moreover, non-selective opioid antagonists, such as naltrexone and naloxone suppress consumption of preferred food, but have smaller effects on non-preferred foods further underlying that opioid signalling is involved in determining food preference (Cooper, 1983; Le Merrer et al., 2009). In agreement, naltrexone reduced sucrose intake in rats previously exposed to a consummatory contrast paradigm, and only for the relatively more valuable sucrose solution (Taha et al., 2006). Further studies, demonstrated that opioid signalling in the NAc strongly modulates flavour-based food choice. Thus, intra-NAc DAMGO (MOR antagonist) increased, while intra-NAc naltrexone decreased, the chocolate flavourbased food consumption highlighting the role of opioid signalling in taste-induced enhancement of food intake (Woolley et al., 2006). In agreement, intra-NAc DAMGO and naltrexone respectively increased and decreased the conditioning flavour preferences as measured by a sensory-specific satiety paradigm used to devalue specific flavours in rats (Woolley et al., 2007b). In contrast, intra-NAc U50488 (KOR agonist) decreased sensory-specific satiety (Woolley et al., 2007a). Finally, NAc and ventral pallidal circuits have been involved in the regulated DAMGO-induced food intake. The 
ventral pallidum, a primary target of NAc efferents, has an important role in normal feeding behaviour since its pharmacological inactivation suppresses baseline and NAc DAMGO-induced consumption. NAc and ventral pallidum circuits seems to converge on a common downstream target that regulates food intake (Taha et al., 2009).

DOR KO animals also decreased active operant responses during the time-out period, which is an indirect measure of impulsive-like behaviours. Interestingly, opiate addicts tend to display impulsivity and behavioural disinhibition particularly in the decisionmaking domain (Clark et al., 2006; Verdejo-Garcia et al., 2007). MOR activity may be positively correlated with impulsivity traits in humans and mice (Love et al., 2009; Olmstead et al., 2009), whereas the opposite implication of DOR in impulsivity and inhibitory control has been reported (Olmstead et al., 2009). Thus, mice lacking DOR appeared more impulsive, in terms of premature responses, than wild-type controls when trained to respond for sucrose in a signalled nose-poke task (Olmstead et al., 2009). In contrast, our results suggest that DOR-deficient mice decrease food-seeking behaviour and impulsive behaviour promoted by the repeated operant training to obtain palatable food. This discrepancy could be due to the different experimental conditions used in terms of rewards, time of sessions and water restriction. Indeed, the previous study found increased impulsivity in response for sucrose solution in session lasting 40 min and when the access to water was restricted for $2 \mathrm{~h}$ per day. These conditions could probably alter inhibitory mechanisms, making these mice incapable of refraining from doing an anticipated response. Conversely, a more recent study suggests that the pharmacological activation of DOR increased locomotor activity, although did not increase the rate of non-reinforced lever pressing for sucrose (Befort et al., 2011), which would suggest that the effect of DOR in impulsive-like behaviour in an operant paradigm could be independent of changes in locomotion. This study provides further 
advances in understanding neuroadaptations related to transition from impulsivity to compulsivity that may promote eating disorders and highlights the relevance of DOR as a potential therapeutic target in these diseases. In this line, previous studies described the involvement of DOR in the reinforcing effects of drugs of abuse (Le Merrer et al., 2009), with a diminished release of DA extracellular levels in the NAc of DOR knockout mice in response to acute cocaine (Chefer et al., 2004). DOR-deficient mice also showed increased ethanol consumption (Roberts et al., 2001) and increased operant behaviour maintained by nicotine intravenous self-administration, although mainly for lower doses of nicotine (Berrendero et al., 2012) which further underline the potential role of DOR in compulsive related behaviour.

In our study, we observed that mice lacking DOR gained less weigh at the end of the operant training when compared with wild-type animals. In agreement, mice deficient in DOR resist to weight gain after prolonged exposure to high-energy diet (Czyzyk et al., 2012). This was attributed to the ability of this receptor to increase the energy expenditure and thermogenic activity in the brown adipose tissue (Czyzyk et al., 2012). Repeated operant training to obtain palatable food changes the morphology of dendritic spines in the mesocorticolimbic system (Guegan et al., 2013) and leads to a reorganization of synaptic connectivity between neurons (Chklovskii, 2004). Palatable food-induced structural plasticity changes in the reward circuit appear to be a longer time-dependent process than drug-induced structural plasticity changes (Sarti et al., 2007). In our study, structural spine density alterations induced by operant training with chocolate-flavoured pellets were revealed in several brain areas of the reward circuit. These morphological spine modifications represent a neuronal substrate underlying food experience-induced behavioural changes (Butz et al., 2009). These adaptations include changes in spine number and shape induced by the synaptic activity. Indeed, the density 
of dendritic spines usually indicates the prevalence of excitatory synaptic inputs into a particular neuron (Lai and Ip, 2013). In this line, an increase of total spine density was found in main rewarding brain areas, such as PFC, HCP and NAc shell of wild-type trained to obtain chocolate pellets when compared with naïve and DOR KO mice. In agreement with previous studies (Guegan et al., 2013), this increased spine density might be responsible for the abnormal behavioural responses observed in wild-type mice trained to obtain palatable pellets, including enhanced seeking-behaviour and related impulsivity. In contrast, reduced spine density was observed in the PFC and HPC of DOR KO mice trained to obtain chocolate pellets with respect to trained wildtype mice. These differences could be attributed in part to the effect of the operant training in the absence of DOR since wild-type trained mice revealed significant modifications in spine density when compared with the non-trained groups. Changes in the total spine density were not observed in the NAc core of DOR KO and wild-type trained mice, underlying that NAc shell and core are involved in different aspects of food seeking behaviour, as previously reported (Peciña and Berridge, 2005). Thus, NAc shell encodes pleasurable sensation derived from food consumption (Baldo and Kelley, 2007), while the NAc core is associated with the elaboration of habit learning promoted mainly by conditioned association of natural reward-related stimuli (Everitt and Robbins, 2005). Accordingly, a previous study reported that operant training to obtain palatable food does not significantly alter dendritic spine density in the NAc core of wild-type mice (Guegan et al., 2013).

Changes in the density of specific dendritic spines were also found in the PFC, HCP and NAc shell between genotypes. Operant training to obtain palatable pellets increased stubby spine density in the HCP and filopodia density in the PFC and NAc shell of wild-type mice in comparison to the non-trained groups. Interestingly, opposite 
structural changes were observed in DOR KO mice, in which a reduced level of stubby spines was observed in the PFC and HCP and a decreased filopodia spine density was found in the PFC and NAc shell of these mice with respect to their wild-type littermates trained to obtain chocolate pellets. Stubby and filopodia spines are recognized as immature spines with low stability, easily turned into other spines or eliminated (Kasai et al., 2002). However, filopodia spines seem to be implicated in synaptogenesis mechanisms due to their elongated morphology that can facilitate axodendritic synaptic contacts (Garcia-Lopez et al., 2010). The decreased number of stubby spines in the PFC and HCP of DOR KO mice could be associated to lower spinogenesis or retraction of the existent spines in the memory/learning and inhibitory control circuits and decreased levels of filopodia spines could be associated to a reduction of synaptic connections in the NAc shell of DOR KO mice. In this line, spine shrinkages, retractions and turnover are generally correlated with LTD activity (Lai and Ip, 2013). Therefore, we can hypothesized that the lower spinogenesis and synaptogenesis in DOR KO mice could be related to possible LTD alterations in these brain areas, leading to decreased motivation for food and reduced operant response and impulsive behaviour.

Operant training with palatable food also enhanced the density of mushroom spines in the PFC and NAc core, as well as thin spines in the PFC in wild-type mice trained to obtain chocolate pellets with respect to the non-trained groups. In contrast, a decrease of thin and mushroom large spines was found in the PFC of DOR KO animals. Mushroom spines hold a decreased motility and consequently display structural and functional stability (Kasai et al., 2004) and are ascribed to mature spines (Nimchinsky et al., 2002) able to maintain pre-existing connections and are considered a structural basis for longterm memory (Kasai et al., 2004). Thin spines maintain structural flexibility to enlarge and stabilize into mushroom spines making them candidate for 'learning spines' 
(Bourne and Harris, 2007). The decreased density of these large spines observed in DOR KO mice could wane the strength of specific synapses in the PFC of these animals. As LTP is generally associated with the enlargement and stability of dendritic spines (De Roo et al., 2008), the present finding may suggest a LTP alteration in the cortical circuit of the mutant mice leading to impairment of food-seeking behaviour.

In conclusion, the present study highlights the role of DOR in the reinforcing and motivational properties of food, as well as in impulsive responses associated to foodseeking behaviour by a mechanism that implies structural plasticity changes in the brain reward circuits. Future studies rescuing DOR expression in KO mice in specific brain areas and neuronal subpopulations will allow defining the precise neuroanatomical sites involved in the observed effects. At the present moment, it cannot be discarded that the observed effects can be influenced by developmental and/or compensatory changes due to persistent absence of DOR (Ruehle et al., 2013). This study provides further advances in understanding neuroadaptations related to food-seeking behaviours that may promote eating disorders and highlights the relevance of DOR as a potential therapeutic target in these diseases. 


\section{Funding and Disclosure}

The authors have no conflicts of interest to declare.

\section{Acknowledgements}

This work was supported by the DG Research of the European Commission FP7 (\#HEALTH-F2 2013-602891), the Spanish 'RETICS-Instituto de Salud Carlos III' (\#RD12/0028/0023), the Spanish 'Ministerio de Ciencia e Innovación’ (\#SAF201129864), the Spanish 'Ministerio de Economia y Competitividad' (\#SAF-2014-59648P), the 'Plan nacional sobre drogas' (\#PNSD-2013-5068), the Catalan Government ‘AGAUR-Generalitat de Catalunya’ (\#2009SGR00731 and \#2014-SGR-1547). The FEDER funds support is also acknowledged. S.M. was supported by FI predoctoral fellowship of the Catalan Government; S.N. was supported by CAPES fellowship of the Brazilian Government. We thank Elysia James for invaluable technical assistance in the structural plasticity study.

\section{Authors contribution}

S.M., S.N conducted the behavioural studies. S.M. participated in the experimental design, neuroplasticity studies, interpretation and manuscript writing. E.M-G. participated in the revision of the manuscript. R.M. participated in the experimental design, supervision of experimental studies, interpretation, manuscript writing and funding of the project. 


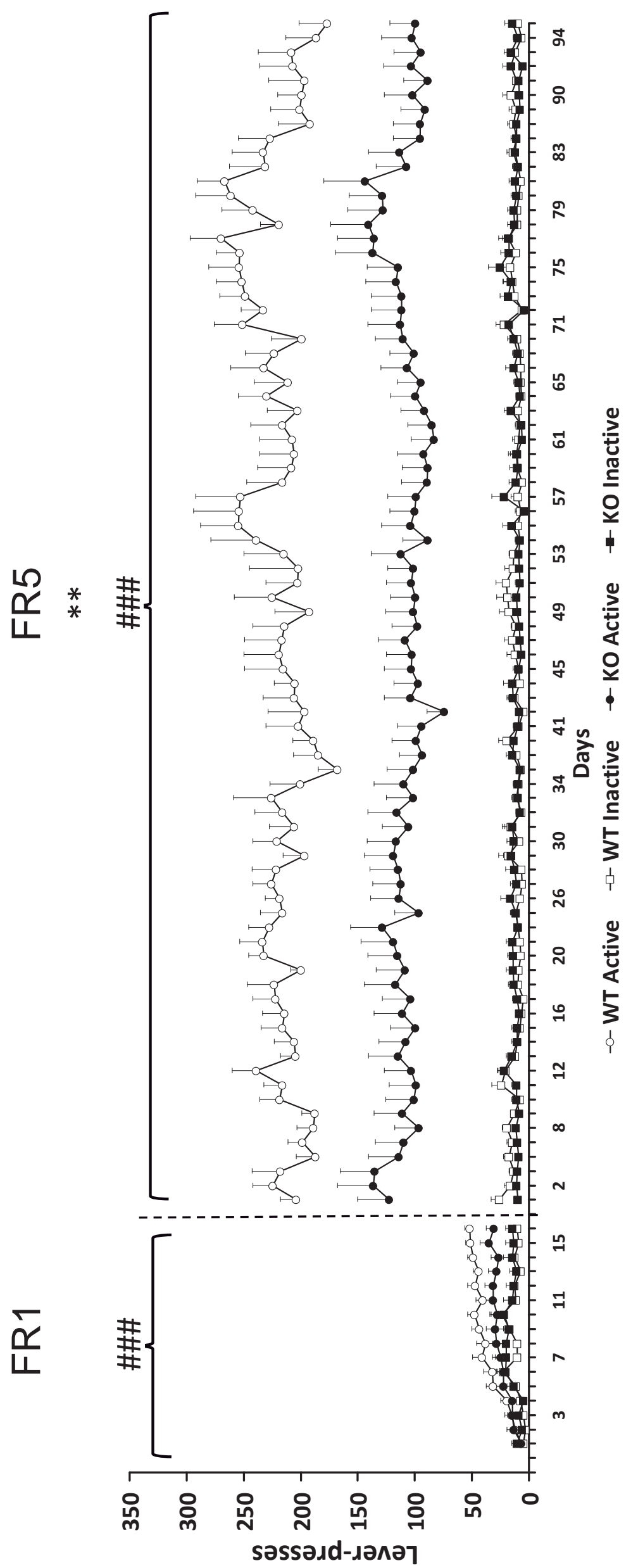

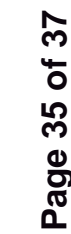



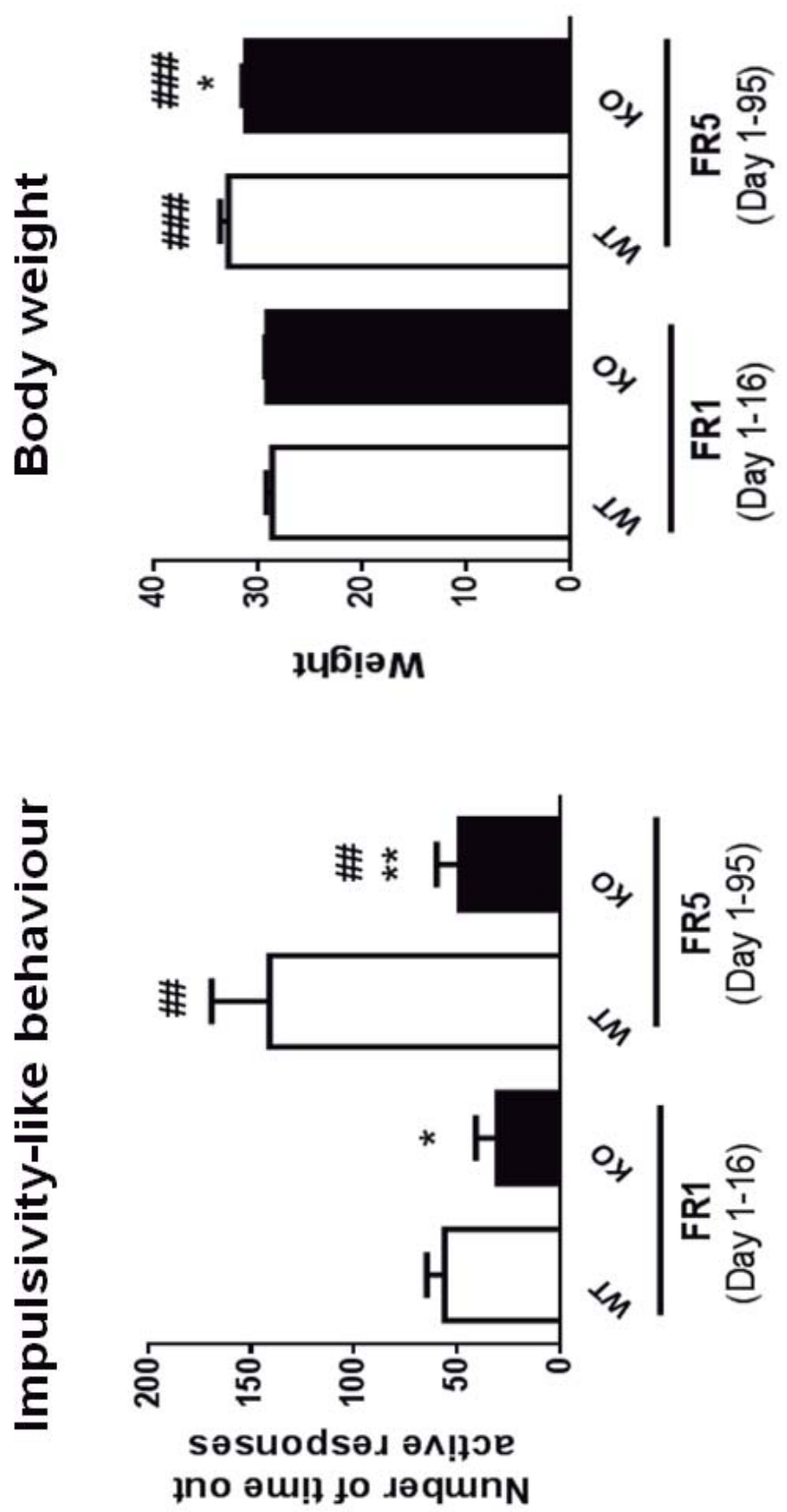

产

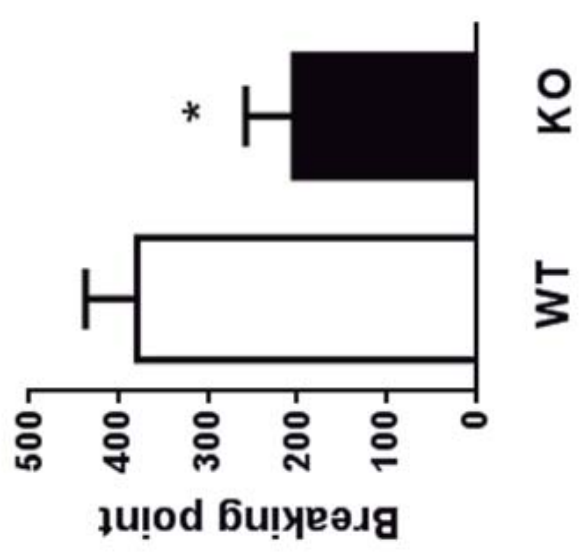


造

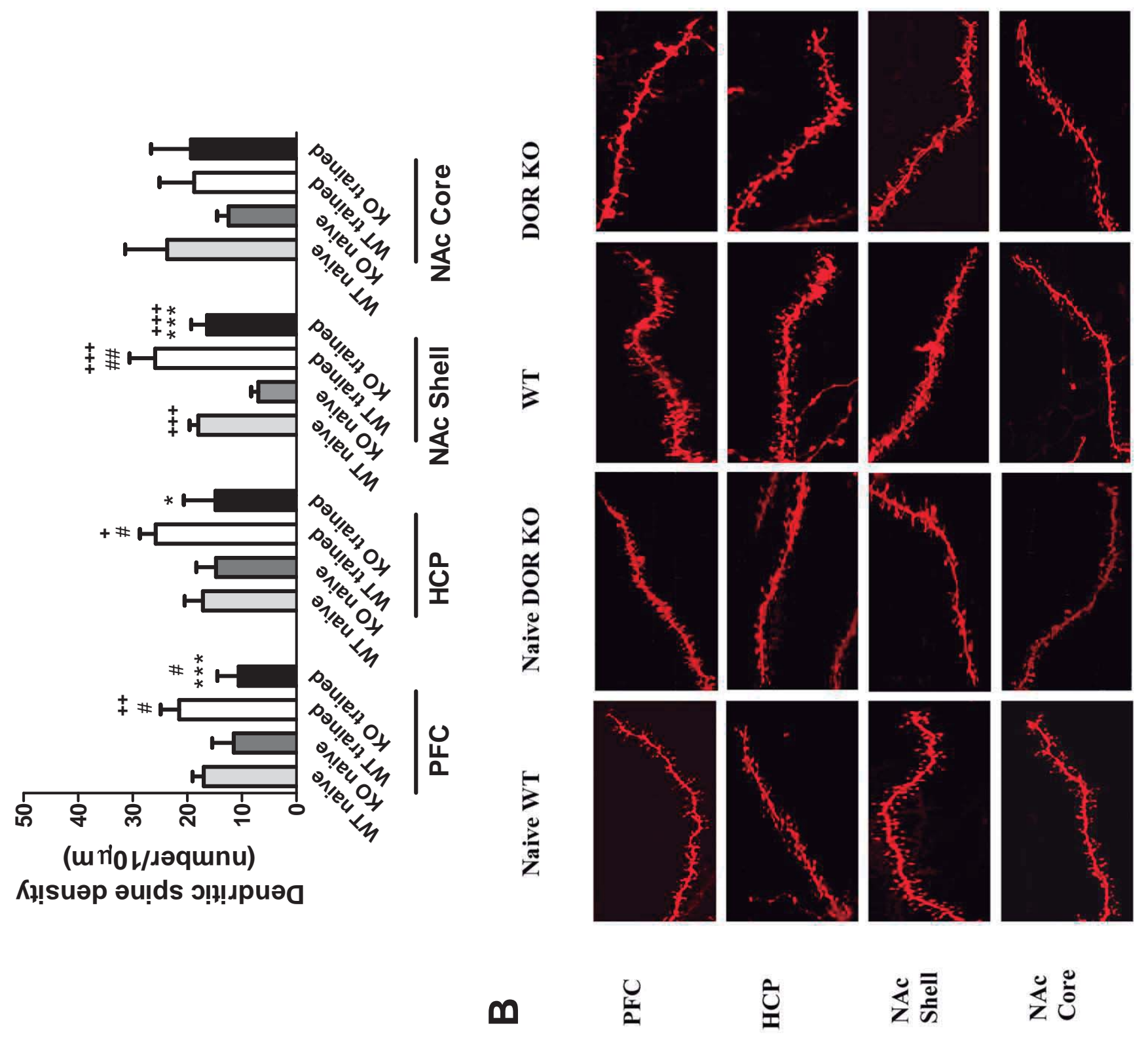

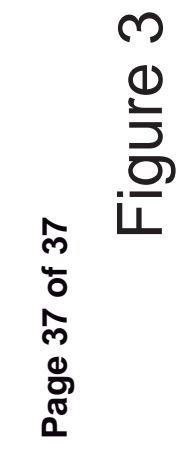



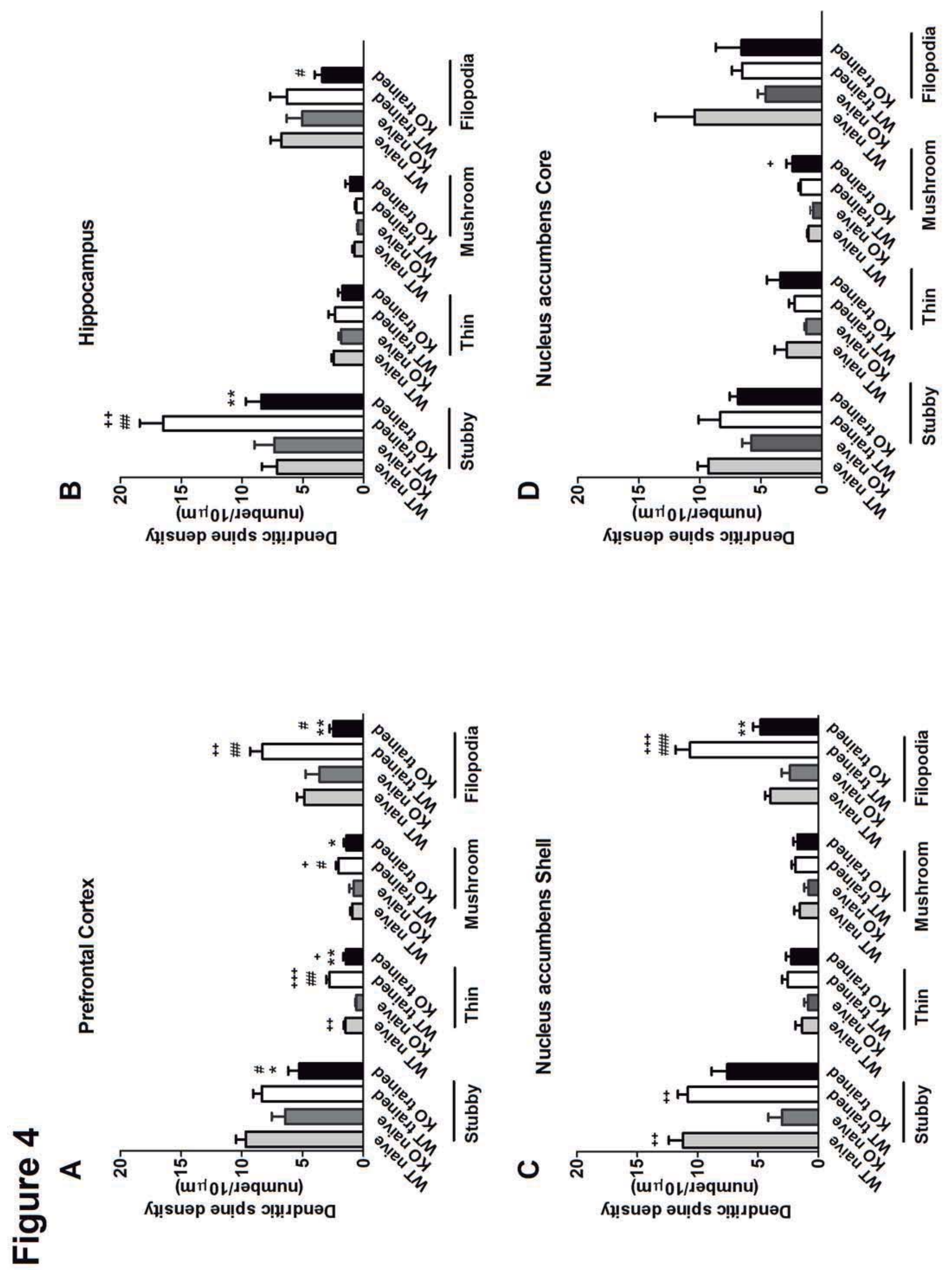


\section{Supporting Information}

Table S1A Normality test

\begin{tabular}{|c|c|c|c|c|c|}
\hline \multicolumn{6}{|c|}{ Kolmogorov-Smirnov } \\
\hline & & \multicolumn{2}{|c|}{ Progressive ratio } & \multicolumn{2}{|l|}{ Time-out } \\
\hline & & $F$-value & $P$-value & $F$-value & $P$-value \\
\hline \multirow[t]{2}{*}{ WT } & FR1 schedule & - & - & $F_{(1,12)}=0.16$ & n.s \\
\hline & FR5 schedule & $F_{(1,12)}=0.20$ & n.s & $F_{(1,12)}=0.20$ & n.s \\
\hline \multirow[t]{2}{*}{ DOR KO } & FR1 schedule & - & - & $F_{(1,14)}=0.06$ & n.s \\
\hline & FR5 schedule & $F_{(1,14)}=0.13$ & n.s & $F_{(1,14)}=0.04$ & $P<0.05$ \\
\hline
\end{tabular}

